\title{
Association between urinary magnesium and glycaemic control in children and adolescents with type 1 diabetes mellitus
}

Filipe Cunha ${ }^{1}$; Juliana Oliveira ${ }^{2}$; Ana Saavedra ${ }^{1}$; Inês Alves ${ }^{2}$; Rita Santos-Silva ${ }^{2}$; Carla Costa ${ }^{3}$; Cíntia Castro-Correia ${ }^{3}$; Manuel Fontoura ${ }^{3}$ 1: Serviço de Endocrinologia, Diabetes e Metabolismo do Centro Hospitalar de São João; 2: Serviço de Pediatria do Centro Hospitalar de São João; 3: Unidade de Endocrinologia Pediátrica do Serviço de Pediatria do Centro Hospitalar de São João

\section{Background}

Hypomagnesaemia is common in diabetic patients particularly in those with poor control. It is unknown whether lower magnesium causes worse glycaemic control or poor control leads to hypomagnesaemia. One possibly mechanism for the latter may be a higher renal magnesium excretion in those patients due to decrease insulin-stimulated magnesium reabsorption.

The relationship between urinary magnesium and glycaemic control is not known. We aimed to study the association between urinary magnesium and glycaemic control in a type 1 diabetes (T1D) pediatric population.

\section{Methods}

Study of a pediatric population with T1D attending the Pediatric Endocrinology Clinic at Hospital São João, Porto, Portugal, between May 2014 and April 2015.

We prospectively included all patients with T1D with an urinary magnesium determination.

Urinary magnesium was measured in a first-morning-void urine sample.

Glycated haemoglobin (HbAlc) was measured in a capilar blood sample using DCA 2000 analyser.

Poor glycaemic control was defined as $\mathrm{HbAlc}>7.5 \%$

Patients with good and poor glycaemic control were compared: $\chi^{2}$ test (with Fisher correction when appropriated), independent samples t test and Mann-Whitney U test.

We studied the correlation between urinary magnesium and HbAlc using Spearman's rank correlation coefficient.

A multivariate logistic regression model was built to study predictors of poor glycaemic control.

\section{Results}

Table 1 - Patients' characteristics

\begin{tabular}{|c|c|c|c|c|}
\hline & All $(n=48)$ & Good control $(n=12)$ & Poor control $(n=36)$ & $\mathbf{p}$ \\
\hline Age, years & 124 & 123 & 124 & 0.63 \\
\hline Male sex, n (\%) & $28(58.3)$ & $6(50.0)$ & $22(61.1)$ & 0.52 \\
\hline T1D duration, months & $88 \quad 43$ & 9343 & $85 \quad 43$ & 0.43 \\
\hline $\mathrm{HbAlc}, \%$ & $8.4 \quad 1.4$ & $\begin{array}{ll}7.0 & 0.4\end{array}$ & $8.9 \quad 1.3$ & $<0.001$ \\
\hline Continuous Subcutaneous Insulin Infusion (CSII), n (\%) & $14(29.2)$ & $7(58.3)$ & $7(19.4)$ & 0.02 \\
\hline Chronic autoimmune thyroiditis, $\mathrm{n}(\%)$ & $11(22.9)$ & $2(16.7)$ & $9(25.0)$ & 0.55 \\
\hline Retinopathy, n (\%) & $1(2.1)$ & $0(0.0)$ & $1(2.8)$ & 1.00 \\
\hline Nephropathy, n (\%) & $7(14.6)$ & $3(25.0)$ & $4(11.1)$ & 0.34 \\
\hline Haemoglobin, g/dL & 14.21 .4 & $13.8 \quad 1.1$ & $14.3 \quad 1.4$ & 0.21 \\
\hline Total Cholesterol, mg/dL & $167 \quad 28$ & $160 \quad 24$ & $170 \quad 29$ & 0.33 \\
\hline $\mathrm{HDL}, \mathrm{mg} / \mathrm{dL}$ & $58 \quad 12$ & $56 \quad 10$ & $58 \quad 12$ & 0.51 \\
\hline $\mathrm{LDL}, \mathrm{mg} / \mathrm{dL}$ & $96 \quad 21$ & $91 \quad 18$ & $98 \quad 22$ & 0.36 \\
\hline Triglycerides, $\mathrm{mg} / \mathrm{dL}$ & $62 \quad 21$ & $66 \quad 19$ & 6121 & 0.52 \\
\hline Creatinine $\mathrm{mg} / \mathrm{dL}$ & $0.54 \quad 0.13$ & $0.53 \quad 0.10$ & $0.55 \quad 0.14$ & 0.76 \\
\hline Ionized Calcium, mEq/L & $2.56 \quad 0.08$ & $2.54 \quad 0.08$ & $2.57 \quad 0.08$ & 0.24 \\
\hline Phosphate, mg/dL & $4.27 \quad 0.62$ & $4.48 \quad 0.54$ & $4.23 \quad 0.64$ & 0.43 \\
\hline Serum magnesium, $\mathrm{mEq} / \mathrm{L}$ & $1.53 \quad 0.10$ & $1.54 \quad 0.09$ & $1.53 \quad 0.11$ & 0.91 \\
\hline Urinary magnesium, $\mathrm{mEq} / \mathrm{L}$ & $8.4(5.3-12.3)$ & $5.6(3.1-7.7)$ & $9.4(6.8-12.4)$ & 0.01 \\
\hline Microalbuminuria, $\mathrm{mg} / \mathrm{g}$ creatinine & $6.65(2.70-10.98)$ & $9(3.8-12.7)$ & $4.8(2.7-9.5)$ & 0.34 \\
\hline
\end{tabular}

Table 2 - Correlation between urinary magnesium (UMg) and serum magnesium, glycaemic control and microalbuminuria

\begin{tabular}{clcccc}
\hline & HbA1c & $\begin{array}{c}\text { T1D } \\
\text { duration }\end{array}$ & $\begin{array}{c}\text { Micro- } \\
\text { albuminuria }\end{array}$ & $\begin{array}{c}\text { Serum } \\
\text { magnesium }\end{array}$ \\
\hline \multirow{2}{*}{ UMg } & rho & 0.30 & -0.17 & 0.28 & 0.07 \\
& p & 0.04 & 0.26 & 0.05 & 0.65 \\
\hline
\end{tabular}

Table 3 - Predictors of poor glycaemic control - Logistic regression multivariate model

\begin{tabular}{lcc}
\hline & Odds Ratio (CI 95\%) & p \\
\hline Age, per year & $0.95(0.68-1.31)$ & 0.75 \\
T1D duration, per month & $0.97(0.94-1.00)$ & 0.04 \\
CSII & $0.06(0.008-0.45)$ & 0.006 \\
UMg, per mEq/L & $\mathbf{1 . 4 0}(\mathbf{1 . 0 5}-1.85)$ & $\mathbf{0 . 0 2}$ \\
\hline
\end{tabular}

\section{Conclusions}

Urinary magnesium is an independent predictor of poor glycaemic control.

Per each $\mathrm{mEq} / \mathrm{L}$ increase of urinary magnesium there is a $40 \%$ higher risk of poor glycaemic control. 